\title{
Modelling multifractal properties of cryptocurrency market
}

\author{
V. Derbentsev ${ }^{1}$, L. Kibalnyk ${ }^{2}$, Yu. Radzihovska ${ }^{3}$ \\ ${ }^{1}$ Department of Information Technologies in Economics, Kyiv National Economic University named after Vadym Hetman, Kyiv, \\ Ukraine. \\ ${ }^{2}$ Department of Economics and Business Modelling, Cherkasy Bohdan Khmelnytsky National University, Cherkasy, Ukraine. \\ ${ }^{3}$ Department of Enterprise Economics, Accounting and Auditing, Cherkasy Bohdan Khmelnytsky National University, Cherkasy, \\ Ukraine.
}

\begin{abstract}
The paper focuses on the study of the effect of long memory and the analysis of the multifractal properties of the time series of the most capitalized cryptocurrencies for the period from 2010 to 2018. To do this, the Hurst exponent is calculated by both $R / S$ analysis and the Detrended Fluctuation Analysis being more stable in the case of non-stationary time series. Our results show that time series of cryptocurrencies to be persistent during almost the whole study period that do not allow accepting the hypothesis concerning the efficiency of the cryptocurrency market. We also found that (i) time series became anti-persistent during the periods of market crisis phenomena and turbulence; (ii) the Hurst exponents showed significant fluctuations about the value of 0.5 . In addition, we conduct a multifractal analysis of cryptocurrency time series that allows us to assess the state and stability of the market.The calculated spectrum of multifractality shows that the cryptocurrency market comes out of a crisis state, since the width of the multifractality spectrum has the maximum value for all cryptocurrencies.
\end{abstract}

\section{Corresponding Author:}

Vasily Derbentsev,

Department of Information Technologies in Economics,

Kyiv National Economic University named after Vadym Hetman,

54/1 Prospect Peremogy, 03057, Kyiv, Ukraine,

Email: derbv@kneu.edu.ua

\section{Introduction}

During its existence, in less than a decade, the cryptocurrencies, primarily, Bitcoin [1] have overcome all the records on the growth of exchange rate and capitalization level, while demonstrating significant fluctuations. Thus, according to Coinmarketcap, [2] the total market capitalization of the cryptocurrency market was approximately $\$ 15.6$ billion at the beginning of 2017; it grew to almost $\$ 800$ billion at the beginning of 2018; and it was $\$ 135.9$ billion on December 2, 2018. The current level of this market capitalization is in line with the capitalization of such companies as Morgan Stanly (\$ 97 billion) and Goldman Sachs Group (\$ 91.7 billion) according to Forbs ranking [3].

Such a rapid, avalanche-like growth and collapse of the cryptocurrency market capitalization in 2018 as a result of a significant fluctuation in their exchange rate (high volatility), on the one hand, and legal unsettledness of the transactions with them in most countries of the world, and consequently, significant risks of investment in cryptoassets, on the other hand, have led to heated discussions about their place and role in the modern economy. The problem of the role and prospects of widespread introduction of cryptocurrency is definitely the most acute and controversial today. 
Therefore, the attention of investors and traders, financial analysts and scientists, has significantly grown to the study of the properties of the cryptocurrency market. As Google Trends statistics showed [4], the terms of Bitcoin and Blockchain ranked among the top 10 searches of this system in 2017.

In particular, the spotlight issues are the determination of factors affecting the exchange rate of digital currencies, whether they are of fundamental value and what is waiting for them in the future, etc. For example, the well-known economists, Nobel Prize Laureates in Economics Paul Krugman, Joseph E. Stiglitz and Robert J. Schiller consider cryptocurrencies, primarily, bitcoin, to be extremely unstable and therefore, in their opinion, the rapid growth of their exchange rate is a typical financial "bubble" [5].

A thorough analysis of the recent research on such aspects of functioning and properties of the cryptocurrency market as the stages of its formation and evolution, the features of functioning, the study of efficiency, legal regulation, etc., was carried out in [6].

Our analysis of literary sources shows that most empirical studies on the cryptocurrency market focus on the following aspects:

(i) the study of efficiency (Efficient Market Hypothesis, EMH) [7]-[10];

(ii) market testing for the presence of speculative bubbles [11]-[16];

(iii) analysis and forecast of price dynamics and volatility [17]-[20].

Recently, modern methods digital signal processing and nonlinear dynamics have been actively introduced for the study of financial time series, in particular, wavelet analysis, fractal and multifractal analysis, entropy analysis, recurrent diagrams and Lyapunov's exponent, etc. [21]-[25].

However, unlike other financial assets (stock price, commodity price, exchange rate, interest rate et al.), studied by a number of empirical researches (See, e.g., [22]-[25]; and literary review in them), the analysis of nonlinear properties of time series of cryptocurrencies is paid much less attention to. At the same time, most papers focus on the analysis of Bitcoin.

Thus, Lahmiri and Bekiros [26] determined chaos in the dynamics of Bitcoin prices during both the period of slow growth and exponential growth with the help of entropy analysis and the calculation of Lyapunov's exponent.

Soloviev and Belinskij [27] showed the efficiency of using recurrent and entropy analysis for the construction of predicting indicators of crisis phenomena on the cryptocurrency market.

Several studies have recently been published concerning the diagnosis of long memory on cryptocurrency market ([7], [10], [28]- [31]). The analysis of Bitcoin time series in Urquhart, [10]; Yonghonga, et al., [31] showed that Hurst statistics was more than 0.5 for all the studied time periods, i.e., the Bitcoin time series was persistent.

However, the additional analysis of Hurst statistics on price and volatility of Bitcoin using Detrended Fluctuation Analysis (DFA) conducted by Bariviera, et al., [29], found that by 2014, this series was persistent, but after this period, these statistics fluctuated within 0.5, being consistent with EMH.

In addition to Bitcoin, such cryptocurrencies as Ripple, Litecoin and DAH were studied using R/S analysis in the work of Caporale, et al., [30]. The meaning of Hurst's statistics was within the range of $H=0.6 \div 0.63$.

The purpose of our research is to test the long memory of Bitcoin and other most capitalized cryptocurrencies, particularly, Ethereum, Bitcoin Cash, EOS, Ripple, Litecoin, Stellar Lumens and DAH, by $R / S$ analysis and DFA, and to study the multifractal properties of the investigated time series by a window procedure using Multifractal-DFA.

\section{Materials and methods}

\subsection{Long-range dependence and EMH}

Long-range dependence is a property that describes the correlation structure of a time series of higher order. In case the series has a long memory, there is dependence even between the observations that are remote in 
time. Since the length of memory creates nonlinear dependence in the first moments of distribution in the model for the medium level and, as a rule, generates a component being potentially suitable for prediction in the dynamics of a series, its presence in the series of profitability of financial assets, raises doubts in the efficiency of the financial market.

According to EMH proposed by Fama [32], the price of an asset in an efficient market should be described by a random walk process, in which each price change does not depend on the previous values, i.e., it has no memory. Due to the fact that the length of memory implies the presence of significant auto-correlation between remote observations, its presence leads to the fact that the past values of the series can help in predicting future values.

Since the length of memory is a special form of nonlinear dynamics, its presence raises doubts about the application adequacy of linear models that requires the necessity of developing new nonlinear models for assessing financial assets to consider the behavior associated with the presence of long memory.

The stationary process $X_{t}$ is a process with long memory if there is a valid number $\alpha, 0<\alpha<1$, and a constant $c>0$, for which the condition is fulfilled:

$$
\lim _{k \rightarrow \infty} \frac{\rho_{k}}{c^{*} k^{-\alpha}}=1,
$$

where $\rho$ - autocorrelation function, $k$ - lag number.

That is, autocorrelations of long memory process satisfy the following asymptotic ratio: $\rho_{k} \sim c^{*} k^{-\alpha}$ when $k \rightarrow \infty$. Thus, the reduction of autocorrelation is slow, according to the hyperbolic law. This hyperbolic decline of autocorrelations is opposed to their exponential decrease, which is characteristic for processes with short memory (ARMA): $\rho_{K} \leq c^{*} a^{k}$, where $c$ - positive constant and $0<a<1$.

There are some alternative approaches to determine of long memory and estimating the fractal time series structure, in particular, R/S analysis suggested by Hurst, [33], and modified Mandelbrot, [34], for the processing of economic time series; standard fluctuation analysis and Detrended Fluctuation Analysis (DFA), proposed by Peng, [35]; Multifractal-DFA (MF-DFA) [36], [37].

\section{2. $R / S$ analysis}

The method of $R / S$ analysis is definitely the most widespread evaluation of long memory in time series, but it is unstable for non-stationary time series (see, for example [38] - [40]). In particular, $R / S$ analysis provides biased estimates for Hurst statistics, when the studied series: (i) contains short-term dependencies; (ii) is nonstationary; (iii) is heterogeneous, i.e., the series contains a heterogeneous sample.

Depending on the value of Hurst exponent $H$, we get three types of time series as shown in Table 1 . Therefore, we use both the standard $R / S$ for estimating the local Hurst exponent and the DFA using a sliding window algorithm.

Table 1. Classification of times eries by the value of the Hurst exponent

\begin{tabular}{|c|c|c|}
\hline $\begin{array}{l}\text { Anti-persistent } \\
\text { (return to the medium value) }\end{array}$ & $\begin{array}{l}\text { Random (stochastic, Wiener } \\
\text { noise, intermediate state) }\end{array}$ & Persistent (trend stable) \\
\hline $0<H<0.5$ & $H=0.5$ & $0.5<H<1$ \\
\hline
\end{tabular}

\section{3. $D F A$ method}

DFA is used to determine (mono-) fractal scaling properties and long-time correlations in noisy nonstationary time series. However, many economic (biological, medical, etc.) objects do not show simple monofractal scaling behavior that can be determined by one coefficient. In some cases, there is a crossover on time scales $s_{x}$, that separates models with different behaviors, i.e., long-time correlations on small time scales $s<<s_{x}$ and correlations of another kind or uncontrolled behavior on a larger scales $s>>s_{x}$. 
Moreover scaling behavior may be more complex, and there are different values of scaling coefficients for different parts of the sequence (for example, for the first and second halves of the sequence). There are even more complicated cases when one-on-one sets with different fractality are considered. It is necessary for such cases to calculate the plurality of scaling coefficients for a complete description of the object behavior. A multifractal analysis of the detrended fluctuations is used on that.

\section{4. $M F$-DFA method}

In general, the MF-DFA procedure consists of five steps proposed Kantelhardt et al. [37]. The first three steps are basically identical to the standard DFA procedure proposed by Peng, [35].

Let $x_{k}$ be a sequence of length $N$ that does not have a large number of zero values (compact support).

Step 1.A profile (accumulation) is determined:

$$
Y(i) \equiv \sum_{k=1}^{i}\left(x_{k}-\bar{x}\right), i=\overline{1 . . N} \text {. }
$$

Subtracting the medium $\bar{x}$ is optional, as it can be done later by detrending in the third step.

Step 2. The profile $Y(i)$ is divided into $N_{s}=\operatorname{int}(N / s)$ segments (sequences) of identical length $s$ that do not overlap. Since the total length of the $N$ sequence does not often divide evenly into s, the remainder at the end of the sequence that is smaller than the window width is discarded. To account for the rejected part and thus to use all the elements of the sequence, the above procedure repeats also from the end of the sequence. Thus, $2 N_{s}$ sequences will be obtained together.

Step 3. For each of the $2 N_{s}$ subsequences, the local trend is calculated using the least squares method. Then the deviation is determined:

$$
F^{2}(v, s)=\frac{1}{s} \sum_{i=1}^{s}\left(Y((v-1) s+i)-y_{v}(i)\right)^{2}
$$

for each segment $v, v=\overline{1 . . N}$ and

$$
F^{2}(v, s)=\frac{1}{s} \sum_{i=1}^{s}\left(Y\left(N-\left(v-N_{s}\right) s+i\right)-y_{v}(i)\right)^{2}
$$

for each $v=\overline{N_{s}+1 . .2 N_{s}}$.

$y_{v}(i)$ is an interpolating polynomial on the $v$ segment. Linear, quadratic, cubic polynomials, or polynomials of higher order are used for interpolation (corresponding methods are called DFA1, DFA2, DFA 3, etc.).

Since the removal of the time series occurs by subtracting the polynomial values from the real values of the series, the DFA of different orders, respectively, differ in their ability to seize the trend in a series. In the MFDFA of $m$-order, profile trends of $m$-order (or, respectively, $(m-1)$-order for the output time series) are deleted. Thus, the comparison of the results of the DFA for different orders is used to obtain the type of trend in the initial time series.

Step 4. The average for all subsequences is found to obtain the function of the fluctuations of the $q$ order:

$$
F_{q}(s)=\left(\frac{1}{2 N_{s}} \sum_{v=1}^{2 N_{s}}\left(F^{2}(s, v)\right)^{q / 2}\right)^{1 / q} .
$$

where, generally speaking, the value of the $q$ variable can be arbitrary, with the exception of zero.

The standard DFA method is obtained for $q=2$. To determine how the timeline $s$ influences on the generalized dependence $F_{q}(s)$ on $q$ at different values of $q$, it is necessary to repeat steps 2-4 for different time scales $s$. It is clear, $F_{q}(s)$ will increase with increasing $q$. Naturally, $F_{q}(s)$ also depends on the order of the DFA method $m$. According to the construction, $F_{q}(s)$ is defined only for $s \geq m+2$ values. 
Step 5. The scaling behavior of the fluctuation function is determined by analyzing the $F_{q}(s)$ dependence on $q$ in the double logarithmic scale. If the $x_{i}$ sequence has long-term correlations, $F_{q}(s)$ increases with increasing $s$ according to the power law

$$
F_{q}(s) \cong s^{h(q)} \text {. }
$$

In general, $h(q)$ coefficient must depend on $q$.For stationary time series, $h(2)$ is identical to the Hurst exponent. Thus, $h(q)$ function can be considered a generalized Hurst exponent.

The monofractal time sequence, $h(q)$ depends on $q$, thus the scaling behavior of $F^{2}(v, s)$ deviations is the same for all $v$ segments and the averaging procedure will give the same values of the scaling coefficient for all segments of the sequence. Only in the case when the scales of small and large fluctuations are different, $h(q)$ dependence will be noticeable on $q$ : if positive values of $q$ are considered, $v$ segments with large values of $F_{s}^{2}(v)$ (e.g., large deviations from the corresponding interpolation polynomials) will dominate in the mean value of $F_{q}(s)$.

Thus, for positive values of $q, h(q)$ describes the scaling behavior of segments with large fluctuations. Large fluctuations are usually characterized by smaller scaling coefficients of $h(q)$ for multifractal series. On the contrary, for negative values of $q, v$ segments with small deviations of $F_{s}^{2}(v)$ will dominate in the mean value of $F_{q}(s)$. Thus, for negative values of $q, h(q)$ describes the scaling behavior of the segments with small fluctuations, which are usually characterized by a larger scaling coefficient.

However, the MF-DFA method can only determine the positive generalized Hurst exponents of $h(q)$, and becomes inaccurate for strongly anti-correlated signals when $h(q)$ is close to zero. In such cases, a modified MF-DFA analysis is used. A simpler way to analyze such data can be the integration of the time series before doing the MF-DFA procedure (finding the accumulations). Hence, the simple finding of the sum in (5), which describes the profile of the initial data, is replaced by a double finding of the sum:

$$
\tilde{Y}(i)=\sum_{k=1}^{i}(Y(k)-\bar{Y})
$$

After this, the procedure of the MF-DFA is done, described above, and as a result, a generalized fluctuation function is obtained:

$$
\tilde{F}_{q}(s) \cong s^{\tilde{h}(q)}=s^{h(q)+1} .
$$

Thus, the scaling behavior can be precisely determined even when $h(q)$ is close to zero (but more than -1 ) for some values of $q$. We should note that $\tilde{F}_{q}(s) / s$ corresponds to $F_{q}(s)$ in (6). If the average value is not deduced from in each step, such a sum will, moreover, give a quadratic trend in $\tilde{Y}(i)$ profile. In this case, it is necessary to use, at least, MF-DFA of the second order to remove such an artificial trend.

The $f(\alpha)$ spectrum of multifractality (singularity) is obtained as a result of applying the following (9):

$$
\alpha=\tau^{\prime}(q), f(\alpha)=q \alpha-\tau(q) .
$$

There is a correlation between $\tau(q)$ and the generalized Hurst exponent:

$$
\tau(q)=q h(q)-1
$$

Linear behavior of $\tau(q)$ means the monofractality of the time series, while the nonlinear - multifractal.

\section{Results and discussion}

\subsection{Data set}

For analysis, we selected the time series of the daily quotations of 8 most capitalized cryptocurrencies from the date of their output to stock exchange on the 4-Nov-2018, according to Coinmarketcap, [2] (Table 2). The 
studied time series of $X(t)$ indices of cryptocurrencies (cryptocurrency quotes to \$ USA) were converted to logarithmic returns, or profitability:

$$
G(t)=\ln X(t+\Delta t)-\ln X(t) \cong \frac{X(t+\Delta t)-X(t)}{X(t)},
$$

where $\Delta t$ is a time interval of delay, which is equal to the 1 st day in our case.

\section{2. $R / S$ analysis}

The results of calculating the local Hurst exponent by $R / S$ using Multifractal Analysis MatLab Toolbox are given in Table 2. All the obtained values of the Hurst exponent can be seen to fall into the range of 0.5 $<H<1.0$. Namely, in fact, the time series can be attributed to persistent, i.e., trend-resistant, to a greater or lesser extent. Trend-resistant behavior, or persistence power, increases with the approach of the Hurst exponent $H$ to 1 . The closer the value of $H$ to 0.5 , the noisier the series is and the less pronounced its trend is. Thus, the most trend-resistant cryptocurrencies were ETH and LTC, and the least trend-resistant - XRP.

Table2. Calculated values of the Hurst exponent $H$ for the most capitalized cryptocurrencies

\begin{tabular}{lllll|l}
\hline & Cryprocurrencies & $\begin{array}{l}\text { Market cap, US \$ } \\
\text { billionon } \\
\text { 4-Nov-2018 }\end{array}$ & Data Start From & $H$ & $\begin{array}{c}H, \\
\text { Caporale, } \text { et } \\
\text { al., [30] }\end{array}$ \\
\hline BTC & Bitcoin & 110,526 & 16-Jul-2010 & 0.751 & 0.59 \\
ETH & Ethereum & 20,591 & 6-Aug-2015 & 0.833 & \\
XRP & Ripple & 18,239 & 21-Jan-2015 & 0.659 & 0.64 \\
BCH & Bitcoin Cash & 8,605 & 31-Jul-2017 & 0.667 & \\
EOS & EOS & 4,809 & 28-Jun-2017 & 0.739 & \\
XLM & Stellar Lumens & 4,471 & 17-Jan-2017 & 0.681 & \\
LTC & Litecoin & 3,008 & 23-Oct-2013 & 0.788 & 0.63 \\
DASH & DASH & 1,300 & 8-Feb-2014 & 0.712 & 0.60 \\
\hline
\end{tabular}

For comparison, Table 2 also shows the results of the estimation of the Hurst exponent, calculated by Caporale, et al., [30], for the period from the beginning of the cryptocurrency operation to 27-Oct-2017. One can see that the persistence of such cryptocurrencies like BTC, LTC and DASH increased during the last year. This can be explained by the fact that a clear downward trend of their exchange rate value was formed in 2018.

\subsection{DFA method}

In addition, we calculated the Hurst exponent $H$ by DFA method. These results, along with the price dynamics (exchange rate), are shown in Fig.1.

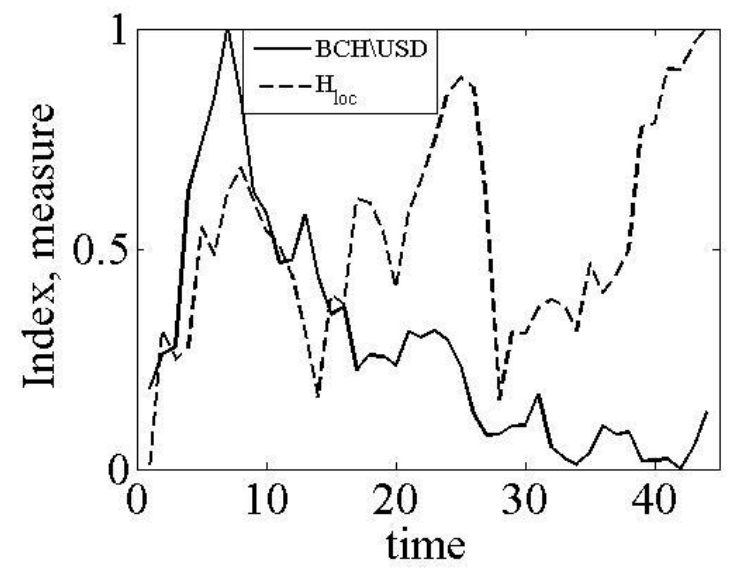

a)

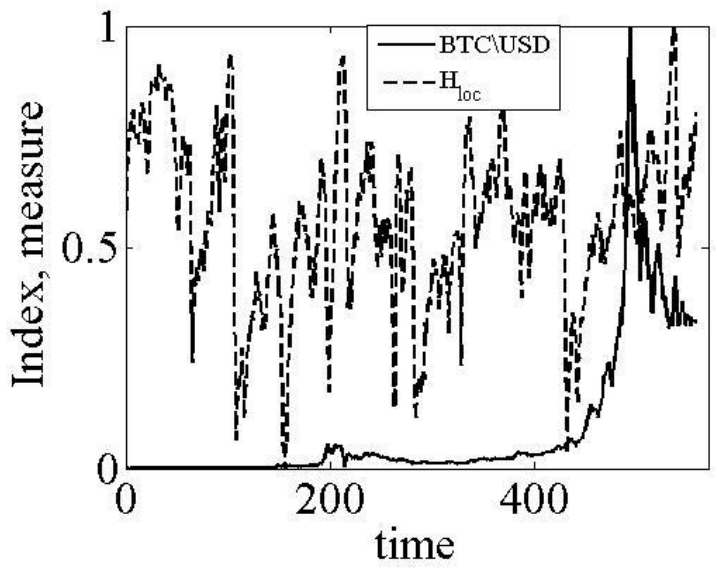

b) 


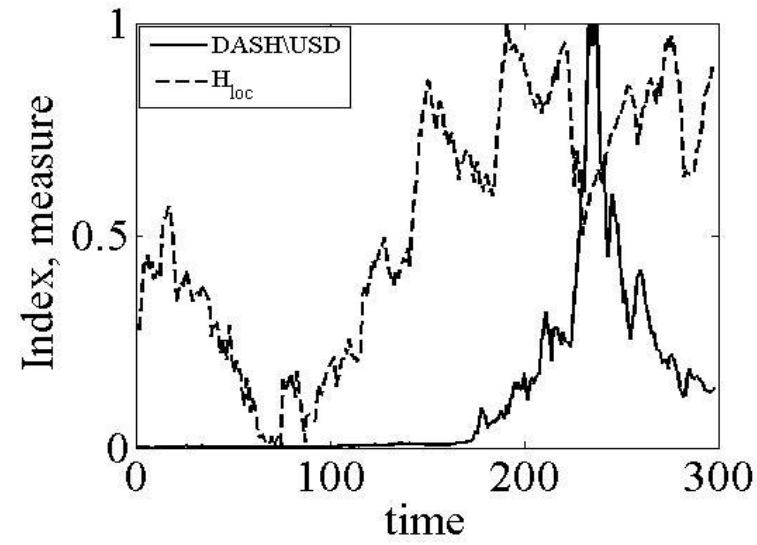

c)

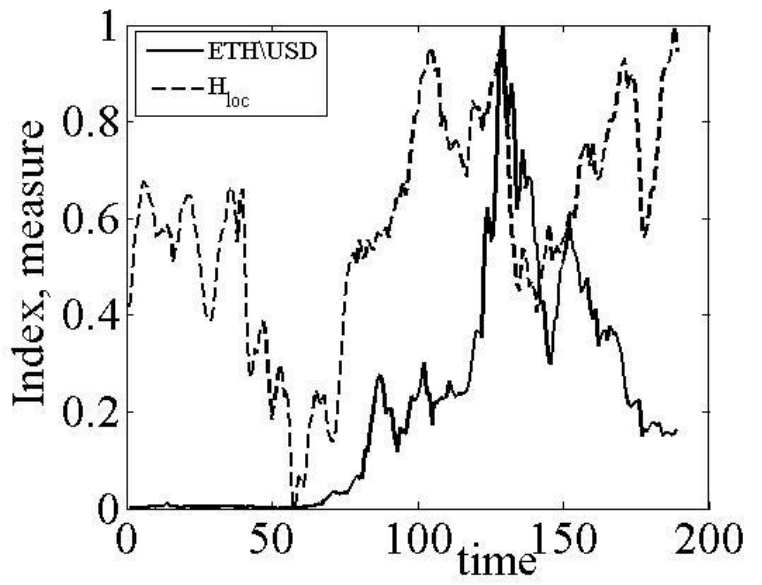

e)

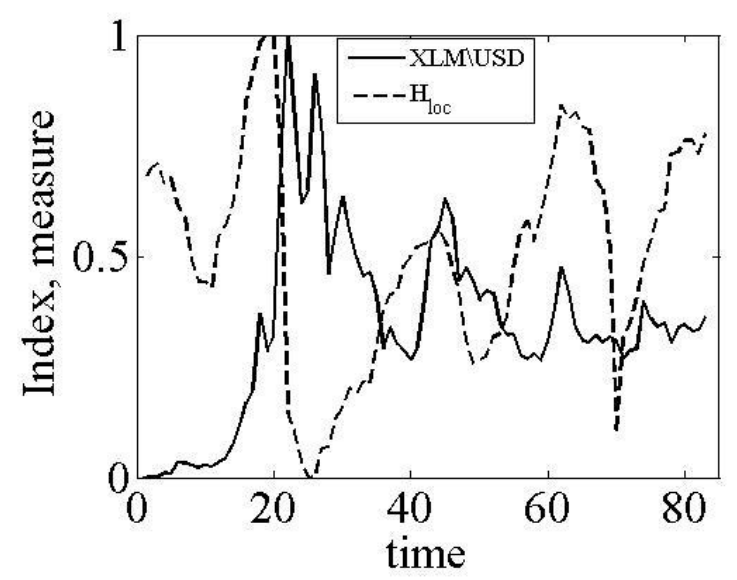

g)

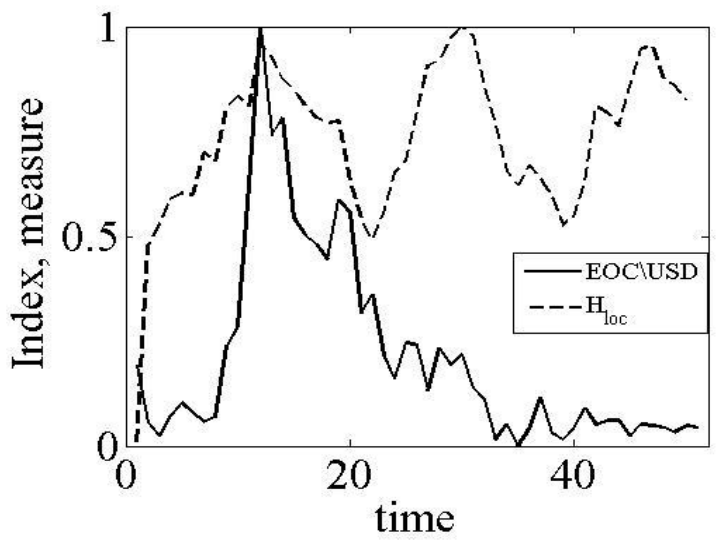

d)

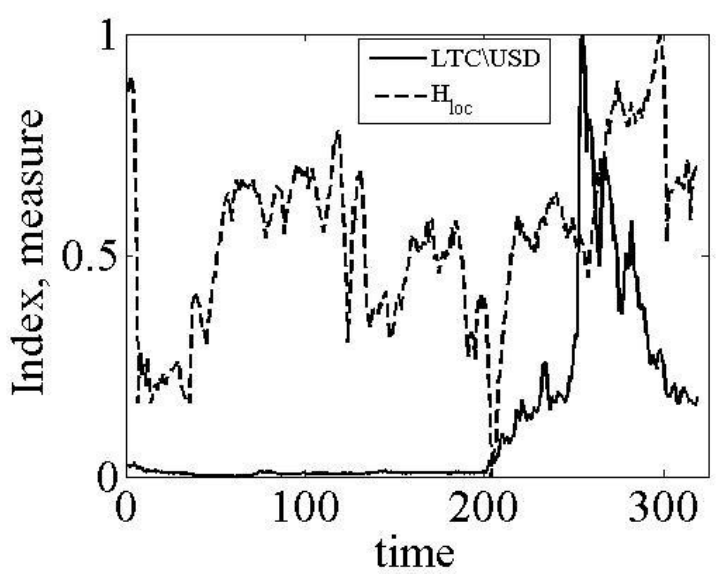

f)

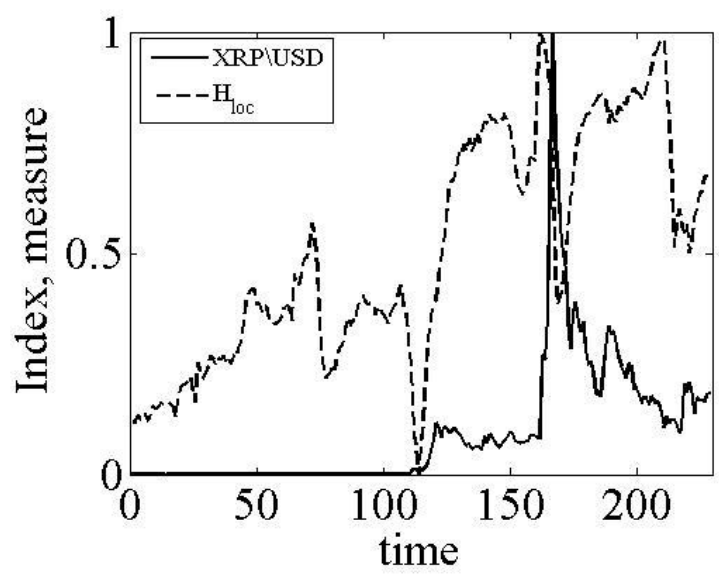

h)

Figure 1. Comparative dynamics of cryptocurrency pair index with local Hurst exponent:

a) BCH/USD, b) BTC/USD, c) DASH/USD, d) EOS/USD; e) ETH/USD; f) LTC/USD; g) XLM/USD; h) XRP/USD.

The calculations were made using a sliding window with the following initial data: the width of the window is 250 points, the step is 5 points. That is, the Hurst exponent is calculated for the first 250 observations, and then sliding window is shifted by 5 observations and is calculated again for the next 250 observations until the end of the sample is reached.

The analysis of Fig.1 allows concluding that the Hurst exponent, calculated by DFA, is sensitive to changes in the cryptocurrency market;at the same time, it shows a tendency to a sharp decline in the periods of crisis phenomena for the most investigated cryptocurrencies. Although most series are persistent during the period 
under study, the series become anti-persistent for such currencies as BTC, BCH, LTC, XLM, XRP in periods of crisis phenomena and increased turbulence in the market; the Hurst exponent shows significant fluctuations near the value of 0.5 , and the price dynamics becomes trend-unstable and unpredictable.

Our results are consistent with Bariviera, et al., [29] who also used DFA for both returns and volatility of Bitcoin time series.According to their results, the Hurst exponent for the BTC for the period from 18.08.2011 to 2.02.2017 was too volatile $H=0,42 \div 0.72$. However, in contrast to their results, we did not find a steady trend for the Hurst exponent up to 0.5 for BTC after 2014, that would be in the case of EHM.

\section{4. $M F-D F A$ method}

To analyze the current market condition, we conducted a dynamic MF-DFA of the time series studied, the results of which are shown in Fig. 2. It shows the change in the average value of the width of the spectrum of multifractality and the average value of the spectrum width in time.

We should note that the studied series have a wide spectrum of multifractality. If multifractalityis treated as a time series behavior, this spectrum indicates a tendency to hold the trend. The width of the spectrum begins to decrease before the crisis, and it reaches its minimum width before the collapse of the index. After the collapse of the index, there is a gradual return of the spectrum width of multifractality to its original value.
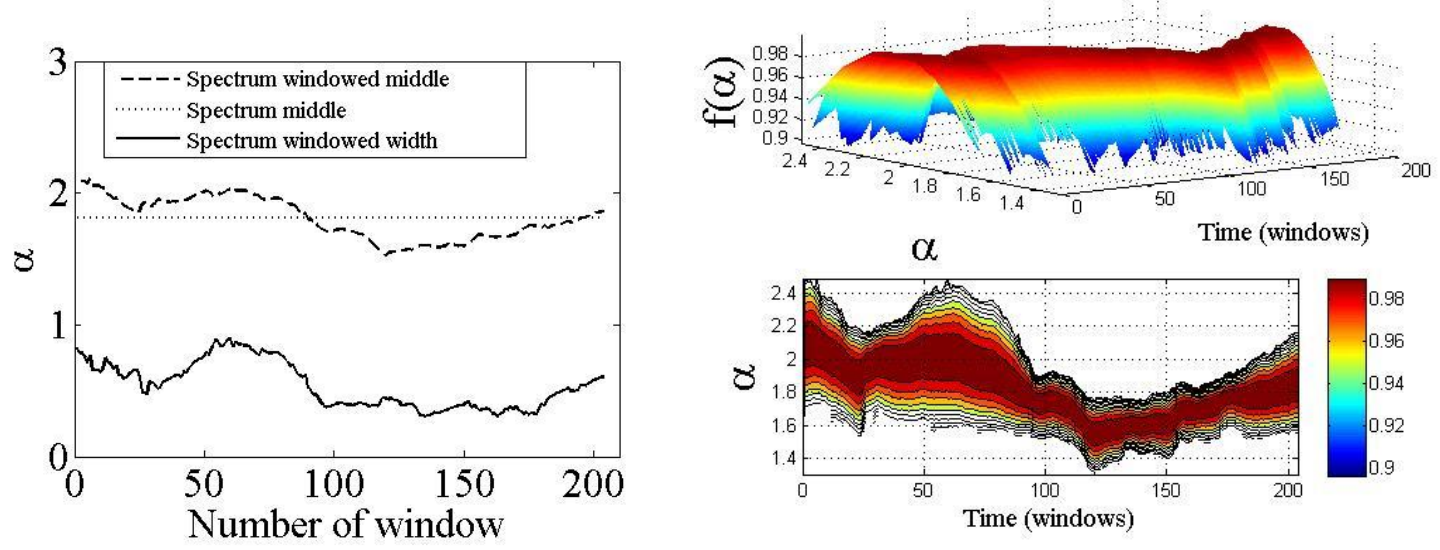

a)
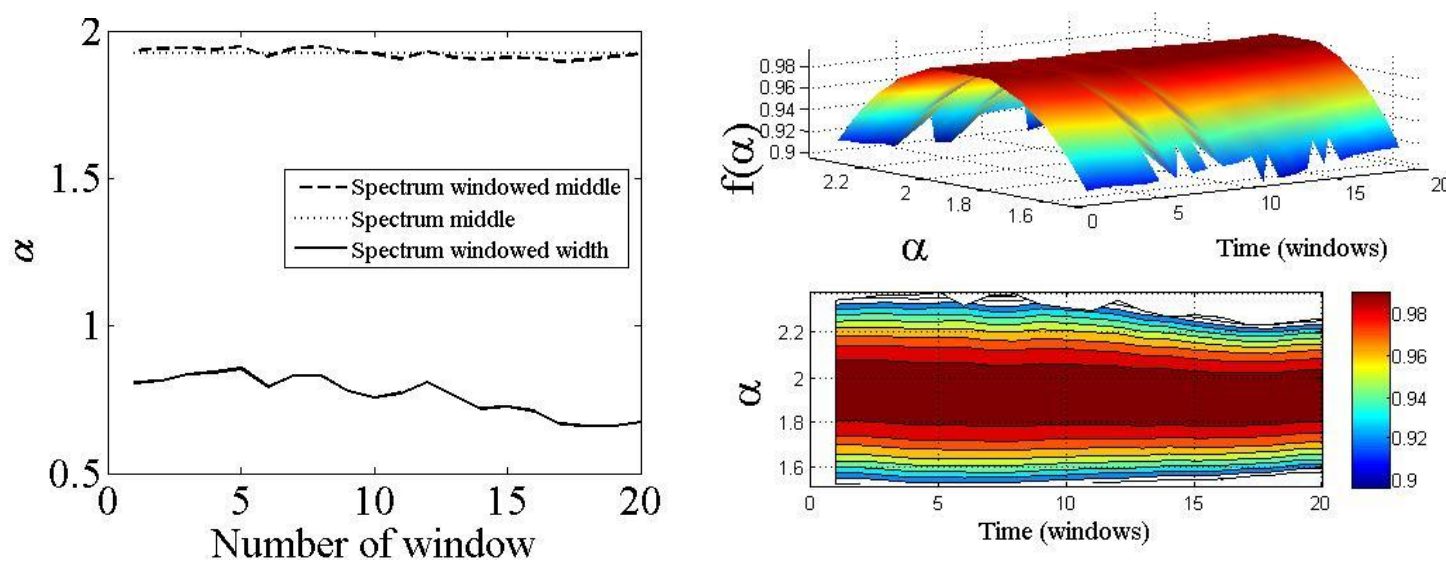

b) 

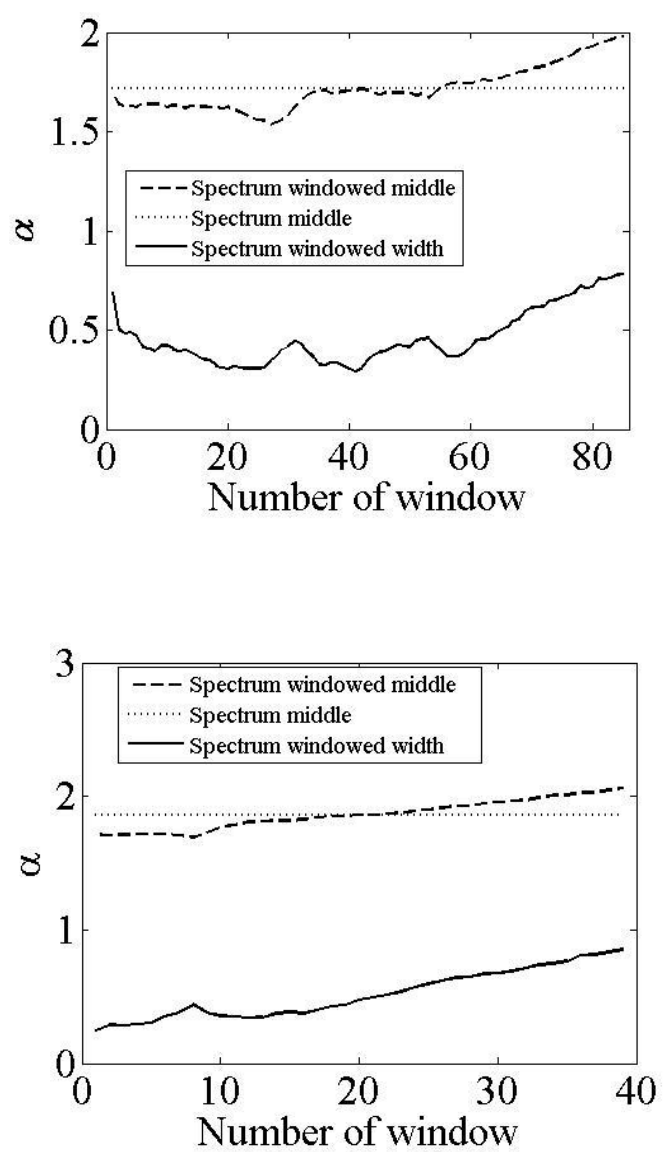
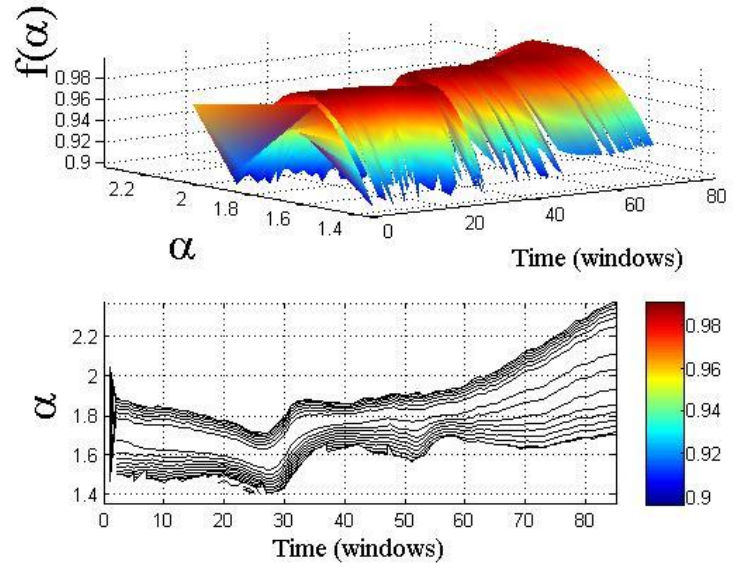

c)
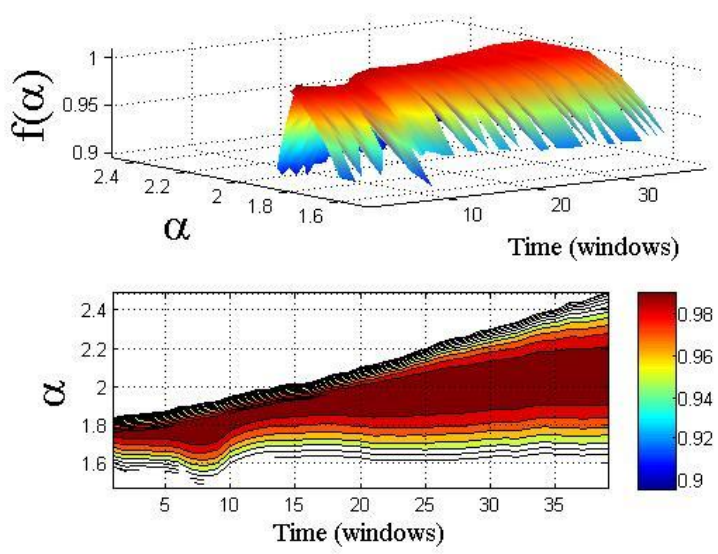

d)

Figure 2. Multifractality spectrum dynamics for cryptocurrency pairs:

a) BTC/USD, b) ETH/USD, c) LTC/USD, d) XRP/USD.

This can be explained by the fact that after the crisis (and the spectrum width of multifractality increases only in this period), the system goes into an unstable state, as a result of which system agents begin to look for the way out of the current state for the system.

The analysis of Fig. 2 shows that the cryptocurrency market is out of crisis state at present, since, as we can see, the spectrum width of multifractality for all cryptocurrencies has the maximum value. At the same time, this indicator clearly demonstrates previous crisis periods (the spectrum of multi-fractality has narrower width). We can observe that the most vulnerable were cryptocurrencies of BTC, LTC, and XRP.

\section{Conclusion}

In this paper, we analyzed the time series of the most capitalized cryptocurrencies in order to diagnose longrange dependence. To do this, we used both standard $R / S$ analysis and DFA, which is more robust, and allows us to evaluate the dynamic properties of the Hurst exponent. The results obtained show that during the study period, most of the time series of cryptocurrencies were persistent, indicating the availability of long memory.

However, during periods of crisis and market turbulence, the series become anti-persistent, the Hurst exponent shows significant fluctuations near the value of 0.5 , and price dynamics becomes trend-unstable and unpredictable.

In addition, we conducted the Multifractal DFA (MF-DFA) and calculated the spectrum of multifractality, which allowed us to assess the state and stability of the market. The studied series have a wide spectrum of multifractality, showing the relaxation and stabilization of the market. 
Thus, on the basis of our analysis, we can identify all the phases of the deployment of crisis phenomena on the cryptocurrency market: pre-crisis state, fall (recession) and relaxation. These states can be determined by the window procedure of multifractal analysis and the calculation of the local Hurst exponent.

In conclusion, it should be noted that our analysis of the fractal properties of cryptocurrencies allows us to make more reasonable decisions for using them as payment instruments or investment assets. Implantation of cryptocurrencies in the e-commerce market allows us to carry out business activities on a global scale [41], to offer enterprises goods and services regardless of spatial and temporal constraints, to reduce the costs of conducting and maintaining operations.

\section{Acknowledgement}

Theauthors express their sincere gratitude to Prof. Vladimir Soloviev for his valuable advice and suggestions on the preparation of this study, and Assist. Prof. Aleksandr Serdiuk for kindly provided for us Multifractal Analysis MatLab Toolbox developed by him.

\section{References}

[1] S. Nakamoto, "Bitcoin: A peer-to-peer electronic cash system," 2009. Avalable online at https://bitcoin.org/bitcoin.pdf. Accessed: 2018-12-02.

[2] Coinmarketcap, Crypto-Currency Market Capitalizations, 2018. Avalable online at https://coinmarketcap.com/currencies/. Accessed: 2018-11-04.

[3] Forbs, 2018. Avalable online at https://www.forbes.com/global2000/\#400c6807335d. Accessed: 2018$12-02$.

[4] Google trends, 2017. Avalable online at https://trends.google.com/trends/yis/2017/GLOBAL/. Accessed: 2018-11-04.

[5] CNBC, "Top Economists Stiglitz, Roubini And Rogoff Renew Bitcoin Doom Scenarios," 2018. Avalable online at https://www.cnbc.com/2018/07/09/nobel-prize-winning-economist-joseph-stiglitzcriticizes-bitcoin.html. Accessed: 2018-12-02.

[6] S. Corbet, B. Lucey, A. Urquhart, and L. Yarovya, "Cryptocurrencies as a financial asset: A systematic analysis, "International Review of Financial Analysis, 2018 (In press).

[7] J. Alvarez-Ramirez, E. Rodriguez, and C. Ibarra-Valdez, "Long-range correlations and asymmetry in the bitcoin market, " Physica A: Statistical Mechanics and its Applications, vol. 492, pp. 948-955, 2018.

[8] S. Nadarajah, and J. Chu, "On the inefficiency of bitcoin," Economics Letters, vol. 150, pp. 6-9, 2017.

[9] R. Selmi, A. Tiwari, and S. Hammoudeh, "Efficiency or speculation? A dynamic analysis of the Bitcoin market," Economics Bulletin, vol. 38, no. 4, pp. 2037-2046, 2018.

[10] A. Urquhart, "The inefficiency of Bitcoin," Economics Letters, vol. 148, pp. 80-82, 2016.

[11] C.Baek, and M. Elbeck, "Bitcoins as an investment or speculative vehicle? a first look, " Applied Economics Letters, vol. 22, no. 1, pp. 30-34, 2015.

[12] B. M. Blau, "Price dynamics and speculative trading in bitcoin, " Research in International Business and Finance, vol. 43, pp. 15-21, 2018.

[13] J. Bouoiyour, and R. Selmi, "What does Bitcoin look like? " Annals of Economics and Finance, vol. 16, no. 2, pp. 449-492, 2015.

[14] A. W.-K. Cheung, E. Roca, and J.-J. Su, "Crypto-currency bubbles: an application of the Phillips? Shi? Yu (2013) methodology on Mt. Gox bitcoin prices," Applied Economics, vol. 47, no. 23, pp. 23482358, 2015.

[15] P. Ciaian, M. Rajcaniova, and D. A. Kancs, "The economics of BitCoin price formation," Applied Economics, vol. 48, no. 19, pp. 1799-1815, 2016.

[16] S. Corbet, B. Lucey and L. Yarovya, "Datestamping the Bitcoin and Ethereum bubbles," Finance Research Letters, vol. 26, no. 9, pp. 81-88, 2018. 
[17] A. Phillip, J.Chan, and S. Peiris, "A new look at Cryptocurrencies," Economic Letters, vol. 163, pp. 69, 2018.

[18] Y. Peng, P. Henrique and M. Albuquerque, "The best of two worlds: Forecasting high frequency volatility for cryptocurrencies and traditional currencies with Support Vector Regression," Expert Systems with Applications, vol. 97, pp. 177-192, 2018.

[19] F. Kjærland, A. Khazal, A. Krogstad, E. Frans, B. Nordstrøm, and A. Oust, "An analysis of Bitcoin's price dynamics," Journal of Risk and Financial Management, vol. 11, no. 63, pp. 1-18, 2018.

[20] M. Balcilar, E. Bouri, R. Gupta, and D. Roubaud, "Can volume predict bitcoin returns and volatility? A quantiles-based approach," Economic Modelling, vol. 64, pp. 74-81, 2017.

[21] N. Prashar, M. Sood, S.Jain, "Semiautomatic Detection of Cardiac Diseases employing Dual Tree Complex Wavelet Transform," Periodicals of Engineering and Natural Sciences, vol. 6, no. 2 , pp. 129-140, 2018.

[22] D. Dechert, Chaos Theory in Economics. Methods, Models and Evidence. Edited by W. Davis, formerly Department of Economics, University of Wisconsin, US, 1996.

[23] V. Derbentsev, O.Serdyuk, V. Soloviev and O. Sharapov, Synergetics and Econophysics Research Methods of Dynamic and Structural Properties of Economic Systems. Cherkasy: Brama-Ukraine, 2010.

[24] J. Ma, and M.Wohar, "Recent advances in estimating nonlinear models with applications in economics and finance," Springer Science, Business Media New York, 2014.

[25] B. LeBaron, Chaos and Nonlinear Forecastability in Economics and Finance. University of Wisconsin - Madison, 1994.

[26] S. Lahmiria, and S. Bekiros, "Chaos, randomness and multi-fractality in Bitcoin market," Chaos Solitons and Fractals, vol. 106(1), pp. 28-34, 2018.

[27] V. Soloviev, and A. Belinskij, "Methods of nonlinear dynamics and the construction of cryptocurrency crisis phenomena precursors," arXiv:1807.05837, 2018. Avalable online at https://arxiv.org/abs/1807.05837. Accessed: 2018-12-02.

[28] Babenko, V.; Perevozova, I.; Mandych, O.; Kvyatko, T.; Maliy, O.; Mykolenko, I., "World informatization in conditions of international globalization: factors of influence, " Global. J. Environ. Sci. Manage., 5(SI): 172-179, 2019. DOI: 10.22034/gjesm.2019.SI.19

[29] A. Bariviera, M. Basgall, W. Hasperue, and M. Naiouf, "Some stylized facts of the Bitcoin market," Physica A: Statistical Mechanics and its Applications, vol. 484, no. 3, pp. 82-90, 2017.

[30] G. Caporale, L. Gil-Alana, and A. Plastun, "Persistence in the cryptocurrency market," Research in International Business and Finance, vol.46, pp. 141-148, 2018.

[31] J. Yonghonga, N. Hea, and R. Weihuab, "Time-varying long-term memory in Bitcoin market," Finance Research Letters, vol. 25, pp. 280-284, 2018.

[32] E. F. Fama, "Efficient capital markets: A review of theory and empirical work, " The Journal of Finance, vol. 25, no. 2, pp. 383-417, 1970.

[33] H. E.Hurst, "Long-term storage capacity of reservoirs," Transactions of the American Society of Civil Engineers, vol. 116, pp. 770-808, 1951.

[34] B. B.Mandelbrot, "Statistical methodology for nonperiodic cycles: From the covariance to R/S analysis," Annals of Economic and Social Measurement, vol. 1, no. 3, pp. 259-290, 1972.

[35] C.-K. Peng, S. Havlin, H. E.Stanley, and A. L. Goldberger, "Quantification of scaling exponents and crossover phenomena in nonstationary heart-beat time series," Chaos: An Interdisciplinary Journal of Nonlinear Science, vol. 5, no. 1, pp. 82-87, 1995.

[36] J. Kantelhardt, Fractal and multifractal time series. In: Encyclopedia of Complexity and Systems Science. New York: Springer, pp. 3754-79, 2009.

[37] J. W. Kantelhardt, S. A. Zschiegner, E. Koscienlny-Bunde, S. Havlin, A. Bunde, and H. E. Stanley, "Multifractal detrended fluctuation analysis of nonstationary time series, " Physica A, vol. 316, no. 1-4, pp. 87-114, 2002. 
[38] Babenko, V., "Formalization of the Model of Management of the Technological Innovations," Proc. 15th Int. Conf. on ICT in Education, Research and Industrial Applications. Integration, Harmonization and Knowledge Transfer (ICTERI2019). Volume II: Workshops, Ed. by Ermolayev, V., Mallet, F., Yakovyna, and oth. (Eds.), Kherson, Ukraine, June 12-15, 2019. Pp. 595-602. CEUR-WS.org, Vol. 2393, 2019, online, http://ceur-ws.org/Vol-2393/.

[39] F. Serinaldi, "Use and misuse of some Hurst parameter estimators applied to stationary and nonstationary financial time series," Physica A: Statistical Mechanics and its Applications, vol. 389, no. 14, pp. 2770-2781, 2010.

[40] C. Cheong, "Estimating the Hurst parameter in financial time series via heuristic approaches," Journal of Applied Statistics, vol. 37, no. 2, pp. 201-214, 2010.

[41] V.Babenko, O. Syniavska,"Analysis of the current state of development of electronic commerce market in Ukraine," Technology Audit and Production Reserves, vol. 5, no 4(43), pp.40-45, 2018. DOI: https://doi.org/10.15587/2312-8372.2018.146341

\section{BIBLIOGRAPHY OF AUTHORS}

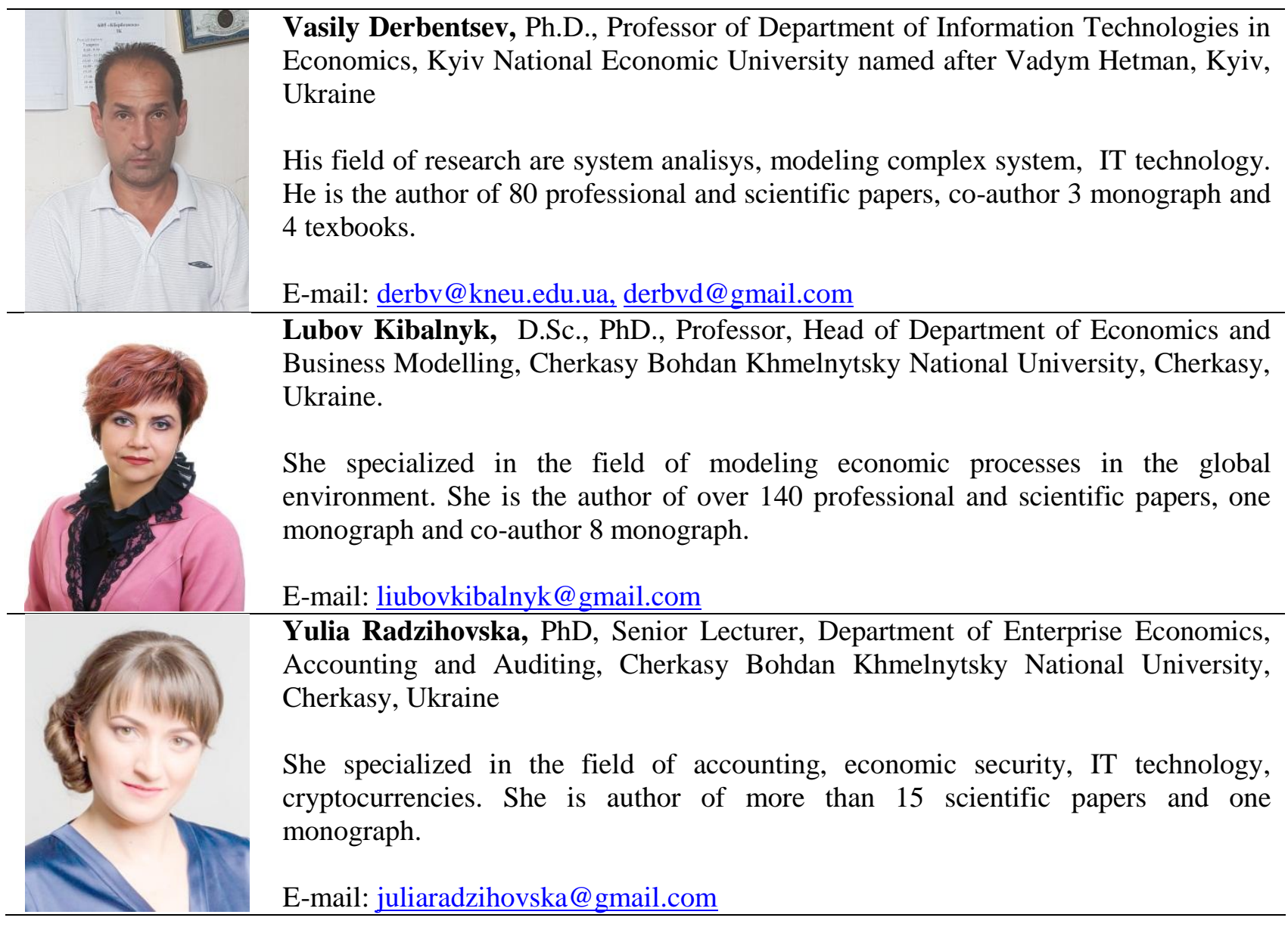

